\title{
ISOPERIMETRIC INEQUALITIES FOR MINIMAL SUBMANIFOLDS IN RIEMANNIAN MANIFOLDS: A COUNTEREXAMPLE IN HIGHER CODIMENSION
}

\author{
VICTOR BANGERT AND NENA RÖTTGEN
}

\begin{abstract}
.
For compact Riemannian manifolds with convex boundary, B. White proved the following alternative: Either there is an isoperimetric inequality for minimal hypersurfaces or there exists a closed minimal hypersurface, possibly with a small singular set. There is the natural question if a similar result is true for submanifolds of higher codimension. Specifically, B. White asked if the nonexistence of an isoperimetric inequality for $k$-varifolds implies the existence of a nonzero, stationary, integral $k$-varifold. We present examples showing that this is not true in codimension greater than two. The key step is the construction of a Riemannian metric on the closed four-dimensional ball $B^{4}$ with the following properties: (1) $B^{4}$ has strictly convex boundary. (2) There exists a complete nonconstant geodesic $c: \mathbb{R} \rightarrow B^{4}$. (3) There does not exist a closed geodesic in $B^{4}$.
\end{abstract}

\section{INTRODUCTION}

If $D$ is a two-dimensional Riemannian disc with locally convex boundary $\partial D$ and if there is no closed geodesic in $D$, then there is a constant $C>0$ such that every geodesic segment in $D$ has length at most $C$. An equivalent formulation of this fact is: If there exists a nonconstant geodesic $c: \mathbb{R} \rightarrow D$, then $D$ contains a closed geodesic. This fact is due to Birkhoff, cf. [2, VI. 10], and played a role in the proof that there exist infinitely many closed geodesics on every Riemannian 2-sphere, cf. [1] and [5].

In arbitrary dimensions, an analogous result has been proven by B. White 8 , Theorem 2.1] in the codimension one situation, i.e. when geodesics are replaced by minimal hypersurfaces. As part of the opening colloquium of the collaborative research center SFB/Transregio 71 in Freiburg, April 2009, B. White lectured on this result, and posed the question if there could be a version of the result that is not restricted to the codimension one case, see also [ 8 , Remark 2.8].

Here, we construct a Riemannian metric $g$ on the closed four-dimensional ball $B^{4}$ such that $\partial B^{4}$ is strictly convex and such that $B^{4}$ carries a complete geodesic, but no closed geodesic. Actually one would expect that such an example exists already on the closed 3-ball. We believe that this is the case, but our construction would be considerably more complicated.

Date: December 23, 2010.

2000 Mathematics Subject Classification. 49Q20, 53C22, 49Q05, 53C42.

Key words and phrases. Riemannian manifolds, geodesics, isoperimetric inequalities, stationary varifolds. 
Now we explain how this can be used to answer B. White's question [8, Remark 2.8], that explicitly asks:

Let $N$ be a compact, $k$-convex Riemannian manifold containing a nonzero, stationary $k$-varifold. Does this imply that $N$ contains an integral stationary $k$-varifold?

For more details on this question see Section 4

Taking the Riemannian product of an arbitrary closed Riemannian manifold $M$ with our example $\left(B^{4}, g\right)$ we obtain a compact manifold $\tilde{M}$ of dimension $\tilde{m} \geq 4$. This $\tilde{M}$ has convex boundary. So $\tilde{M}$ is $k$-konvex for every $k<\tilde{m}$. The product of $M$ with a complete geodesic in $B^{4}$ gives an $(\tilde{m}-3)$-dimensional, immersed, totally geodesic submanifold isometric to $M \times \mathbb{R}$. This implies that there is no isoperimetric inequality for $(\tilde{m}-3)$-dimensional minimal submanifolds in $\tilde{M}$. Hence, from 8 , Theorem 2.3] we know that there exists a nonzero, stationary $(\tilde{m}-3)$-varifold in $\tilde{M}$. Indeed, we can describe explicitly such a varifold $V_{0}$ in $M$, and prove that - up to scale $-V_{0}$ is the only stationary $(\tilde{m}-3)$-varifold in $\tilde{M}$.

From the explicit description of $V_{0}$ we conclude that $V_{0}$ is not rectifiable and, hence, not integral. This gives a negative answer to B. White's question for the case of varifolds of arbitrary dimension, and codimension at least three.

Finally we sketch the idea underlying the construction of the metric $g$ on $B^{4}$. First, we deform the standard metric $g_{0}$ on the ball $B^{4} \subset \mathbb{R}^{4}$ of radius 2 so that all the spheres $S^{3}(\rho) \subset B^{4}$ of radius $\left.\rho \in\right] 0,2$ [ remain strictly convex, except for $S^{3}(1)$ whose second fundamental form vanishes precisely on the vectors tangent to an irrational geodesic foliation $\mathcal{F}$ of the Clifford torus $\mathbb{T}^{2} \subset S^{3}(1)$. This implies that there are no closed geodesics in $B^{4}$ with respect to this metric. Moreover, we achieve that also the second fundamental form of the Clifford torus $\mathbb{T}^{2}$ vanishes in the direction of $\mathcal{F}$. Then the leaves of $\mathcal{F}$ are complete geodesics not only in $\mathbb{T}^{2}$ but also with respect to the metric on $B^{4}$.

\section{Contents}

1. Introduction

2. Convex Distance Functions

3. The Example

4. An answer to a question by Brian White 6

5. Appendix 10

References

\section{Convex Distance Functions}

In this section we will recall some well known facts about geodesics and distance functions. Let $(M, g)$ denote a Riemannian manifold and $i: N \hookrightarrow M$ a submanifold. We will denote the induced metric on $N$ by $g^{N}$. Then a curve $c: I \subset \mathbb{R} \rightarrow N$ is a $g$-geodesic if and only if $c$ is a $g^{N}$-geodesic and the second fundamental form of $N$ vanishes on its tangent vectors.

Now, let $F: N \times(-\varepsilon, \varepsilon) \rightarrow M$ be a normal variation with variational vector field $V=\left.\frac{\partial F}{\partial t}\right|_{t=0}$ along $i=F(\cdot, 0)$. Then, for any tangent vectors $v_{1}, v_{2} \in T_{p} N$, one 
calculates, cf. [3. (1.33)],

$$
\frac{d}{d t}_{\mid t=0}\left(F_{t}^{*} g\right)\left(v_{1}, v_{2}\right)=g\left(\nabla_{v_{1}} V, v_{2}\right)+g\left(v_{1}, \nabla_{v_{2}} V\right),
$$

where $F_{t}: N \rightarrow M$ is defined by $F_{t}(\cdot)=F(\cdot, t)$.

If, additionally, $|V|=1$, it follows from equation (11), that the second fundamental form $h^{N}(\cdot, \cdot)$ of $N$ with respect to $V$ is given by

$$
h^{N}\left(v_{1}, v_{2}\right)=-\left.\frac{1}{2} \frac{d}{d t}\right|_{t=0}\left(F_{t}^{*} g\right)\left(v_{1}, v_{2}\right) .
$$

We will use this fact in the special case where $N$ is a level set of a $C^{\infty}$-function $d$ with $|\operatorname{grad} d|=1$. These functions will be called distance functions, cf. [6, 2.3.1]. Then the restriction of the gradient flow $\Phi_{t}$ to $N$ is a normal variation with variational vector field $V=\operatorname{grad} d$. The gradient of $d$ is contained in the null space of the Hessian $\nabla^{2} d$ and for any $v_{1}, v_{2} \in T_{p} N$ one obtains

$$
\nabla^{2} d\left(v_{1}, v_{2}\right)=g\left(\nabla_{v_{1}} V, v_{2}\right)=-h^{N}\left(v_{1}, v_{2}\right) .
$$

Hence a distance function is a convex function if the second fundamental form (with respect to $\operatorname{grad} d$ ) of any of its level sets is everywhere negative semidefinite. Recall that a $C^{2}$-function $f: M \rightarrow \mathbb{R}$ is convex if one of the following equivalent conditions is satisfied:

- For any geodesic segment $c: I \rightarrow M$ the composition $f \circ c: I \rightarrow \mathbb{R}$ is convex.

- The Hessian $\nabla^{2} f$ is everywhere positive semidefinite.

In particular, we have:

Fact 1. Let $f: M \rightarrow \mathbb{R}$ be a convex function. Then any closed geodesic in $M$ is contained in one of the level sets of $f$. If the second fundamental form of a smooth level set of $f$ is definite at some point, then there is no closed geodesic passing through this point.

Therefore there are no closed geodesics on a manifold that is equipped with a convex distance function, if its Hessian restricted to the tangent spaces of the level sets is everywhere definite.

\section{The ExAMPLE}

Consider the closed standard 4-ball $\left(B^{4}, g_{0}\right)$ with radius 2 and the Clifford torus $\left(\mathbb{T}^{2}, g_{0}^{\mathbb{T}^{2}}\right)$ given by $\{1 / \sqrt{2}(\sin \varphi, \cos \varphi, \sin \theta, \cos \theta) \mid \varphi, \theta \in[0,2 \pi]\}$. The Clifford torus is a flat torus that is isometrically embedded in the standard sphere $S^{3} \subset B^{4}$. The map $(\varphi, \theta) \in \mathbb{R}^{2} \rightarrow \frac{1}{\sqrt{2}}(\sin \varphi, \cos \varphi, \sin \theta, \cos \theta)$ from euclidean $\mathbb{R}^{2}$ to the Clifford torus $\mathbb{T}^{2}$ is a homothetic covering map. The projection to $\mathbb{T}^{2}$ of a family of parallel lines in $\mathbb{R}^{2}$ will be called a geodesic foliation of $\mathbb{T}^{2}$. A geodesic foliation of $\mathbb{T}^{2}$ is called rational if the corresponding family of parallels has rational slope and irrational otherwise. The geodesics of a rational foliation of $\mathbb{T}^{2}$ are all closed, while the geodesics of an irrational geodesic foliation of $\mathbb{T}^{2}$ are all dense on $\mathbb{T}^{2}$.

The metric $g$ that we will define on $B^{4}$ will have the following properties:

(G1) The induced metric $g^{\mathbb{T}^{2}}$ on $\mathbb{T}^{2}$ is the flat one induced by $g_{0}$.

(G2) The function $d: B^{4} \rightarrow[0,2]$ given by the euclidean distance to zero is a convex distance function with respect to the metric $g$. 
(G3) There exists an irrational geodesic foliation $\mathcal{F}$ of $\mathbb{T}^{2}$ such that the following holds for the hessian $\nabla^{2}\left(d^{2}\right)$ with respect to $g:\left.\nabla^{2}\left(d^{2}\right)\right|_{x}$ is positive definite for all $x \in B^{4} \backslash \mathbb{T}^{2}$, and for $x \in \mathbb{T}^{2}$ the nullspace of $\left.\nabla^{2}\left(d^{2}\right)\right|_{x}$ coincides with the tangent line to $\mathcal{F}$ at $x$.

(G4) The second fundamental form of the Clifford torus $\mathbb{T}^{2}$ as a submanifold of $S^{3}$ vanishes on the vectors tangent to the irrational geodesic foliation $\mathcal{F}$ of $\mathbb{T}^{2} \subset S^{3}$.

From (G2), (G3) and equation (3) we conclude

(G3') For any sphere $\left.\left.S^{3}(\rho)=d^{-1}(\rho), \rho \in\right] 0,2\right] \backslash\{1\}$, the second fundamental form $h^{S^{3}(\rho)}$ with respect to $\operatorname{grad} d$ is negative definite, and on $S^{3}=S^{3}(1)$ the zero directions of $h^{S^{3}}$ are precisely the vectors tangent to the irrational geodesic foliation $\mathcal{F}$.

Now we will prove

Proposition 1. Suppose $g$ is a Riemannian metric on $B^{4}$ satisfying conditions (G(1))-(G(4). Then there exists a complete (non-constant) g-geodesic $c: \mathbb{R} \rightarrow B^{4}$, but no closed g-geodesic in $B^{4}$. Moreover, $\partial B^{4}=S^{3}(2)$ is strictly convex.

Proof. Note first that by conditions (G3) and (G4) the geodesics of the irrational foliation are complete $g$-geodesics contained in $\mathbb{T}^{2} \subset B^{4}$, cf. the discussion at the beginning of Section 2. Next we will show that there are no closed $g$-geodesics in $B^{4}$. So, let us assume that there exists a closed $g$-geodesic $c: S^{1} \rightarrow B^{4}$. Using properties (G2), (G3) and Fact 1 we conclude that $c$ lies in the euclidean sphere $S^{3}$ and that $c$ is a leaf of the irrational foliation $\mathcal{F}$ of $\mathbb{T}^{2} \subset S^{3}$. This contradicts our assumption that $c$ is closed.

Now we describe how one can construct a Riemannian metric $g$ on $B^{4}$ that satisfies properties (Gप)-(Gष). We consider the coordinate system

$$
\begin{aligned}
F:] 0,2[\times] 0, \pi / 2\left[\times \mathbb{R}^{2}\right. & \longrightarrow B^{4} \\
(\rho, \psi, \varphi, \theta) & \longmapsto\left(\begin{array}{ccc}
\rho & \cos \psi & \sin \varphi \\
\rho & \cos \psi & \cos \varphi \\
\rho & \sin \psi & \sin \theta \\
\rho & \sin \psi & \cos \theta
\end{array}\right) .
\end{aligned}
$$

For $\rho=1$ and $\psi=\pi / 4$ the coordinates $\varphi$ and $\theta$ describe the Clifford torus, i.e. $\mathbb{T}^{2}=F\left(\{1\} \times\{\pi / 4\} \times \mathbb{R}^{2}\right)$. We denote the induced coordinate vectors on $\operatorname{im}(F):=$ $F(] 0,2[\times] 0, \pi / 2\left[\times \mathbb{R}^{2}\right)$ by $\partial_{\rho}, \partial_{\psi}, \partial_{\varphi}, \partial_{\theta}$. They form a $g_{0}$-orthogonal frame on $\operatorname{im}(F)$, and the metric $g_{0}$ is given in these coordinates by the diagonal matrix

$$
\operatorname{diag}\left(1, \rho^{2}, \rho^{2} \cos ^{2} \psi, \rho^{2} \sin ^{2} \psi\right) .
$$

This shows, in particular, that $\left.F\right|_{\{1\} \times\{\pi / 4\} \times \mathbb{R}^{2}}$ is - up to the constant factor $\frac{1}{\sqrt{2}}$ - an isometric covering map with group of deck transformation $2 \pi \mathbb{Z} \times 2 \pi \mathbb{Z}$. For fixed $\alpha \in \mathbb{R} \backslash \mathbb{Q}$ we consider the vectorfield $Y=\partial_{\varphi}+\alpha \partial_{\theta}$. The restriction of $Y$ to the torus $\mathbb{T}^{2}$ is tangent to an irrational geodesic foliation and the vector field $Z:=\alpha \tan \psi \partial_{\varphi}-\cot \psi \partial_{\theta}$ completes $\partial_{\rho}, \partial_{\psi}$ and $Y$ to an orthogonal frame on $\operatorname{im}(F)$. We define a new metric $g$ on $\operatorname{im}(F)$ by requiring that the vectorfields $\partial_{\rho}$, 
$\partial_{\psi}, Y$ and $Z$ are pairwise $g$-orthogonal and by setting for $x=F(\rho, \psi, \varphi, \theta)$

$$
\begin{aligned}
g\left(\partial_{\rho}, \partial_{\rho}\right)_{\mid x} & =g_{0}\left(\partial_{\rho}, \partial_{\rho}\right)_{\mid x}=1, \\
g\left(\partial_{\psi}, \partial_{\psi}\right)_{\mid x} & =g_{0}\left(\partial_{\psi}, \partial_{\psi}\right)_{\mid x}=\rho^{2}, \\
g(Y, Y)_{\mid x} & =R(\rho, \psi), \\
g(Z, Z)_{\mid x} & =g_{0}(Z, Z)_{\mid x}=\rho^{2}\left(\cos ^{2} \psi+\alpha^{2} \sin ^{2} \psi\right),
\end{aligned}
$$

where the function $R \in C^{\infty}(] 0,2[\times] 0, \pi / 2\left[, \mathbb{R}^{+}\right)$is chosen such that the following conditions are fulfilled:

(R1) $R(\rho, \psi)=\rho^{2}\left(\cos ^{2} \psi+\alpha^{2} \sin ^{2} \psi\right)$,

$$
\text { if }(\rho, \psi)=(1, \pi / 4) \text { or }(\rho, \psi) \in(] 0,2[\times] 0, \pi / 2[) \backslash([1 / 2,3 / 2] \times[\pi / 8,3 \pi / 8])
$$

(R2) $\frac{\partial}{\partial \rho} R(\rho, \psi)>0$ if $(\rho, \psi) \neq(1, \pi / 4)$

(R3) $D R_{\mid(1, \pi / 4)}=0$

For completeness, we will construct such a function $R$ in the appendix. First note that condition (RI) ensures that $g$ coincides with the standard metric $g_{0}$ outside the tubular neighborhood of $\mathbb{T}^{2}$ given by the image of $[1 / 2,3 / 2] \times[\pi / 8,3 \pi / 8] \times \mathbb{R}^{2}$ under $F$. Therefore, the standard metric extends $g$ to a smooth metric on all of $B^{4}$.

Proposition 2. The metric $g$ fulfills conditions (G(1)-(G4).

Proof. First note that condition (G1) follows from condition (R1). Next, our definition of $g$ directly implies that $\partial_{\rho}$ is the $g$-gradient of the euclidean distance $d$ from zero. Hence $d$ is a distance function also with respect to $g$. Thus, by the discussion in Section 2, we can calculate its $g$-Hessian on $\operatorname{im}(F)$ with equations (2) and (3). As grad $\left.d\right|_{\operatorname{im}(F)}=\partial_{\rho}$ commutes with $\partial_{\psi}, Y$ and $Z$, we obtain for $V, W \in\left\{\partial_{\psi}, Y, Z\right\}$

$$
\frac{d}{d t}{ }_{\mid t=t_{0}}\left(\Phi_{t}^{*} g\right)_{\mid p}(V, W)=\frac{d}{d t}{ }_{\mid t=t_{0}} g_{\Phi_{t}(p)}\left(V_{\mid \Phi_{t}(p)}, W_{\mid \Phi_{t}(p)}\right),
$$

where $\Phi_{t}$ denotes the gradient flow of $d$. Remember that on $\operatorname{im}(F)$ the flow lines of $\Phi$ are the $\rho$-coordinate lines. Using the preceding equation and equations (2) and (3) we see that on $\operatorname{im}(F)$ the matrix of the $g$-Hessian of $d$ with respect to the frame $\partial_{\rho}, \partial_{\psi}, Y, Z$ is the diagonal matrix given by

$$
\operatorname{diag}\left(0, \rho, \frac{1}{2} \frac{\partial}{\partial \rho} R(\rho, \psi), \rho\left(\cos ^{2} \psi+\alpha^{2} \sin ^{2} \psi\right)\right)
$$

Now, condition (G3) follows immediately from (R2) and (R/3). Since the metric coincides with the standard metric in a neighborhood of $B^{4} \backslash \operatorname{im}(F)$ and the Hessian of $d$ is positive semidefinite on $\operatorname{im}(F)$, the function $d$ is convex everywhere. So, also condition (G2) is proven. Finally, to prove (G4), we consider the projection $\left.\pi_{\psi}: S^{3} \cap \operatorname{im}(F) \rightarrow\right] 0, \pi / 2[, \quad F(1, \psi, \varphi, \theta) \mapsto \psi$. This provides a distance function with gradient $\partial_{\psi}$ whose gradient flowlines are given by the coordinate lines of $\psi$. Now we calculate the second fundamental form $h^{\mathbb{T}^{2}}$ of $\mathbb{T}^{2}$ in $S^{3}$ with respect to $\partial_{\psi}$, using equation (2). Then $\left[Y, \partial_{\psi}\right]=0$ and condition (R 3 imply:

$$
h^{\mathbb{T}^{2}}(Y, Y)=-\frac{1}{2} \frac{\partial}{\partial \psi} R(1, \pi / 4)=0
$$

This completes the proof. 
Remark 1. The construction above can easily be generalized to balls $B$ of dimension $n \geq 5$. The construction yields a Riemannian metric on $B$ fulfilling properties (G1)-(G4) with the obvious modifications of the dimension.

\section{An answer to a question by Brian White}

As mentioned in the introduction, our example is related to isoperimetric inequalities in Riemannian manifolds. Brian White [8] showed that an isoperimetric inequality holds for minimal hypersurfaces (or -more generally- for codimension one varifolds) in a compact, connected Riemannian manifold $\tilde{M}$ with mean-convex boundary if $\operatorname{dim}(\tilde{M})<7$ and if there does not exist a smooth, closed, embedded minimal hypersurface $N \subset \tilde{M}$ (The same conclusion is true if $\operatorname{dim}(\tilde{M}) \geq 7$, provided one replaces "smooth" by "smooth except for a singular set of Hausdorff dimension at most $\operatorname{dim}(\tilde{M})-7 ")$.

An isoperimetric inequality in higher codimension is obtained in 8 , Theorem 2.3 under the stronger condition, that there does not exist any nonzero, stationary varifold of the same codimension. In this context, Brian White asks (cf. 8, Remark 2.8]), whether the existence of a nonzero, stationary $k$-varifold in a compact, $k$-convex Riemannian manifold $N$ implies the existence of a nonzero, stationary, integral $k$-varifold in $N$. For a brief introduction to varifolds on Riemannian manifolds see [8, Appendix].

In the following Proposition we answer this question in the negative for codimesion larger than 2 . Starting with an arbitrary closed, connected, $m$-dimensional Riemannian manifold $\left(M, g^{\prime}\right)$ we consider the product metric $\tilde{g}=g^{\prime} \oplus g$ on $\tilde{M}=M \times B$, where $B$ is a closed ball of dimension $n \geq 4$ and $g$ a Riemannian metric on $B$ fullfilling (G1)-(G4), cf. Section 3. Then $\partial \tilde{M}=M \times \partial B$ has the following convexity property. The second fundamental form of $\partial \tilde{M}$ with respect to the inward pointing unit normal is positive semi-definite, and its kernel consists of the vectors tangent to the factor $M$. In Proposition 3 we will show that $(\tilde{M}, \tilde{g})$ contains a unique stationary, $(m+1)$-dimensional varifold $V_{0}$ of unit mass, and, in Fact 2, that $V_{0}$ is not rectifiable and, hence, not integral. This provides a negative answer to the question posed in [8, Remark 2.8]. It is easy to see that $(\tilde{M}, \tilde{g})$ does not either admit an isoperimetric inequality for $(m+1)$-dimensional submanifolds with boundary: Denoting, as before, by $c: \mathbb{R} \rightarrow \mathbb{T}^{2} \subset B$ a g-geodesic that is dense on the Clifford torus $\mathbb{T}^{2}$, we consider the totally geodesic submanifolds $M_{n}=M \times c([-n, n])$ in $\tilde{M}$. They satisfy $\operatorname{vol}_{m+1}\left(M_{n}\right)=2 n \operatorname{vol}_{m}(M)$, while $\operatorname{vol}_{m}\left(\partial M_{n}\right)=2 \operatorname{vol}_{m}(M)$. This contradicts the existence of an isoperimetric inequality for $(m+1)$-dimensional minimal submanifolds (with boundary) in $\tilde{M}$.

Remark 2. According to B. White's proof of [8, Theorem 2.3] any limit of the varifolds induced by the $M_{n}$, normalized so as to have mass one, is a non-zero, stationary, $(m+1)$-dimensional varifold. It is easy to see (and follows from Proposition (3) that in our case there is a unique limit varifold and that this is equal to $V_{0}$.

Next we describe the $(m+1)$-varifold $V_{0}$ in $\tilde{M}$ : A general $(m+1)$-varifold in $\tilde{M}$ is a finite Borel measure on the total space of the Grassmann bundle $\pi: G_{m+1}(\tilde{M}) \rightarrow$ $\tilde{M}$. The support of $V_{0}$ is the subset $\tilde{\mathcal{F}}$ of $G_{m+1}(\tilde{M})$ given by

$$
\tilde{\mathcal{F}}=\left\{T_{p} M \times T_{q} \mathcal{F} \mid(p, q) \in M \times \mathbb{T}^{2}\right\},
$$


where $\mathcal{F}$ is the foliation of the Clifford torus $\mathbb{T}^{2} \subset B$ defined in (G3). In particular, $\left.\pi\right|_{\tilde{\mathcal{F}}}$ is one-to-one. Now $V_{0}$ is the pushforward of the normalized Riemannian volume of $M \times \mathbb{T}^{2}$, i.e. $V_{0}=\left(\left.\pi\right|_{\tilde{\mathcal{F}}} ^{-1}\right)_{\#} \operatorname{vol}_{M \times \mathbb{T}^{2}}$.

In particular, the weight measure $\mu_{V_{0}}$ of $V_{0}$ is the normalized Riemannian volume $\operatorname{vol}_{M \times \mathbb{T}^{2}}$ of the $(m+2)$-dimensional submanifold $M \times \mathbb{T}^{2}$. This implies that the $(m+1)$-density of $\mu_{V_{0}}$ is identically zero.

For rectifiable $(m+1)$-varifolds $V$ the weight measure $\mu_{V}$ has an approximate tangent space for $\mu_{V}$ almost every point and hence its $(m+1)$-density is positive $\mu_{V}$-almost everywhere, cf. [7, §15]. Since the $(m+1)$-density of $\mu_{V_{0}}$ vanishes, we conclude

Fact 2. The $(m+1)$-varifold $V_{0}$ is not rectifiable.

Here is the main result of this section.

Proposition 3. Let $(\tilde{M}, \tilde{g})$ and $V_{0}$ be as above. Then $V_{0}$ is stationary, and $V_{0}$ is the only stationary $(m+1)$-varifold of mass one in $(\tilde{M}, \tilde{g})$.

Remark 3. Statement and proof of Proposition 3 include the case $\operatorname{dim}(M)=m=$ 0 . In this case the only stationary, unit mass 1-varifold in $B$ is the stationary, non-rectifiable 1-varifold $V_{0}$ with support on the tangent vectors to the irrational geodesic foliation $\mathcal{F}$ of $\mathbb{T}^{2}$ (see the description of $V_{0}$ above).

We first recall the following well known fact from ergodic theory:

Fact 3. (cf. 44, p. 69]) Suppose $T_{t}$ is the one-parameter group of translations on the standard torus $\mathbb{R}^{2} /(2 \pi \mathbb{Z})^{2}$ given by $\left[\left(x_{1}, x_{2}\right)\right] \mapsto\left[\left(x_{1}+\alpha_{1} t, x_{2}+\alpha_{2} t\right)\right]$ with $\alpha_{1}$ and $\alpha_{2}$ rationally independent. Then the flow $T_{t}$ is uniquely ergodic, i.e. the Lebesgue measure $\mu$ on $\mathbb{R}^{2} /(2 \pi \mathbb{Z})^{2}$ is the - up to scale - unique $T_{t}$-invariant Borel measure on $\mathbb{R}^{2} /(2 \pi \mathbb{Z})^{2}$.

Corollary 1. Let $\bar{Y}$ be the unit vector field on $\mathbb{T}^{2}$ tangent to $\mathcal{F}$, that is given by the normalisation of $\left.Y\right|_{\mathbb{T}^{2}}$, cf. Section 3, and denote by $\varphi_{t}^{\bar{Y}}$ its flow. Then the Riemannian area $\operatorname{vol}_{\mathbb{T}^{2}}$ is the - up to scale - unique Borel measure on $\mathbb{T}^{2}$ that is invariant under $\varphi_{t}^{\bar{Y}}$.

Proof. The norm of the vectorfield $Y=\partial_{\varphi}+\alpha \partial_{\theta}$ is constant on $\mathbb{T}^{2}$, and we denote it by $a=|Y|_{\mathbb{T}^{2}} \mid=\frac{1}{\sqrt{2}} \sqrt{1+\alpha^{2}}$. Now, the covering map $\rho: \mathbb{R}^{2} \rightarrow \mathbb{T}^{2}$, $\rho\left(x_{1}, x_{2}\right)=F\left(1, \frac{\pi}{4}, x_{1}, x_{2}\right)$ induces a diffeomorphism $\tilde{\rho}: \mathbb{R}^{2} /(2 \pi \mathbb{Z})^{2} \rightarrow \mathbb{T}^{2}$ conjugating the irrational linear flow $T_{t}$ from Fact 3 with $\alpha_{1}=\frac{1}{a}$ and $\alpha_{2}=\frac{\alpha}{a}$ to the flow $\varphi_{t}^{\bar{Y}}$. So, by Fact 3 the push-forward $\tilde{\rho}_{\#} \mu$ of the Lebesgue measure $\mu$ on $\mathbb{R}^{2} /(2 \pi \mathbb{Z})^{2}$ is the - up to scale - unique $\varphi_{t}^{\bar{Y}}$-invariant Borel measure on $\mathbb{T}^{2}$. On the other hand, $\tilde{\rho}_{\#} \mu$ equals $\operatorname{vol}_{\mathbb{T}^{2}}$ up to a factor since $\tilde{\rho}$ is a homothety.

We first give a short outline of the proof of Proposition 3. In Step 1 we calculate that $V_{0}$ is indeed stationary, see also Remark 4 In Step 2 and 3 we consider an arbitrary nonzero, stationary $(m+1)$-varifold $V$ in $\tilde{M}$. In Step 2 we show that its support is contained in the set $\tilde{\mathcal{F}} \subset G_{m+1}(\tilde{M})$. This relies on the convexity properties of the spheres $S^{3}(\rho) \subset B$, cf. Section 3 . In the last step, we use the Constancy Theorem [7, 41.2(3)] to prove that the weight measure $\mu_{V}$ of $V$ has a product structure. Then the unique ergodicity of the flow $\varphi_{t}^{\bar{Y}}$ can be used to show that $\mu_{V}$ is indeed proportional to the product measure $\operatorname{vol}_{M} \otimes \operatorname{vol}_{\mathbb{T}^{2}}$. This proves that $V=\lambda V_{0}$ for some $\lambda>0$. 


\section{Proof of Proposition 3:}

Step 1: Here we prove that $V_{0}$ is stationary.

We recall that the vectorfield $\bar{Y}$ (see Corollary 1 for the definition of $\bar{Y}$ ) is parallel, and spans $T_{q} \mathcal{F}$ at every point $q$ of $\mathbb{T}^{2}$. We decompose any vectorfield $X$ on $\tilde{M}$ as a $\operatorname{sum} X(p, q)=X_{q}^{1}(p)+X_{p}^{2}(q)$, where $X_{q}^{1}(p) \in T_{p} M$ and $X_{p}^{2}(q) \in T_{q} B$. So, by the special character of the Levi Civita connection of a Riemannian product, we obtain for every $(p, q) \in M \times \mathbb{T}^{2}$ :

$$
\begin{aligned}
\operatorname{div}_{T_{p} M \times T_{q} \mathcal{F} X} & =\left.\operatorname{div}_{M}\left(X_{q}^{1}\right)\right|_{p}+\left.g\left(\nabla_{\bar{Y}} X_{p}^{2}, \bar{Y}\right)\right|_{q} \\
& =\left.\operatorname{div}_{M}\left(X_{q}^{1}\right)\right|_{p}+\left.\frac{d}{d t}\right|_{t=0} g\left(X_{p}^{2}, \bar{Y}\right) \circ \varphi_{t}^{\bar{Y}}(q),
\end{aligned}
$$

where $\varphi_{t}^{\bar{Y}}$ denotes the flow of $\bar{Y}$. Now the Gauss Theorem and the invariance of the volume of the flat torus under $\varphi_{t}^{\bar{Y}}$, cf. Corollary 1 imply that

$$
\begin{aligned}
\delta V_{0}(X)= & \int_{M \times \mathbb{T}^{2}} \operatorname{div}_{T_{p} M \times T_{q} \mathcal{F}} X d \mu_{V_{0}}(p, q) \\
= & \left.\int_{\mathbb{T}^{2}} \int_{M} \operatorname{div}_{M}\left(X_{q}^{1}\right)\right|_{p} \operatorname{dvol}_{M}(p) \operatorname{dvol}_{\mathbb{T}^{2}}(q) \\
& \quad+\left.\int_{M} \int_{\mathbb{T}^{2}} \frac{d}{d t}\right|_{t=0} g\left(X_{p}^{2}, \bar{Y}\right) \circ \varphi_{t}^{\bar{Y}}(q) \operatorname{dvol}_{\mathbb{T}^{2}}(q) \operatorname{dvol}_{M}(p) \\
= & 0+\left.\int_{M} \frac{d}{d t}\right|_{t=0}\left(\left.\int_{\mathbb{T}^{2}} g\left(X_{p}^{2}, \bar{Y}\right)\right|_{q} d\left(\varphi_{t}^{\bar{Y}}\right)_{\#} \operatorname{vol}_{\mathbb{T}^{2}}(q)\right) \operatorname{dvol}_{M}(p) \\
= & 0 .
\end{aligned}
$$

So $V_{0}$ is stationary.

Now, we consider an arbitrary nonzero, stationary $(m+1)$-varifold $V$ in $\tilde{M}$.

Step 2: First, we prove that the varifold $V$ has support in $\tilde{\mathcal{F}}$.

We consider $f: \tilde{M} \rightarrow \mathbb{R}_{\geq 0},(p, q) \mapsto d^{2}(q)$, where $d(q)$ denotes the (euclidean) distance from $q \in B$ to $0 \in B$, cf. Section 3. Note that (G2) and (G3) imply the following: If $(v, w) \in T_{p} M \times T_{q} B$ then $\nabla^{2} f((v, w),(v, w))>0$ except in the following two cases

- $w=0$, or

- $q \in \mathbb{T}^{2}$ and $w \in T_{q} \mathcal{F}$.

Now suppose $V$ is a stationary $(m+1)$-varifold in $\tilde{M}$. We test $V$ against the vectorfield $X=\operatorname{grad} f$. Then we have

$$
0=\delta V(X)=\int \operatorname{div}_{S} X d V(S)=\iint_{G_{m+1}(\tilde{M})} \operatorname{trace}_{S}\left(\nabla^{2} f\right) d V(S) .
$$

The preceding discussion shows that $\operatorname{trace}_{S}\left(\nabla^{2} f\right)>0$ except if $S \in \tilde{\mathcal{F}}$. Hence $\operatorname{spt}(V) \subset \tilde{\mathcal{F}}$.

Step 3: We show that $\mu_{V}$ equals $\operatorname{vol}_{M \times \mathbb{T}^{2}}$ up to a constant.

First, we prove that for any Borel set $A \subset B$ there exists $c_{A}>0$ such that the Borel measure $\mu^{A}$ on $M$ defined by $\mu^{A}(\cdot):=\mu_{V}(\cdot \times A)$ is given by $c_{A} \cdot \operatorname{vol}_{M}$. 
Note that $\mu^{A}$ can be considered as an $m$-varifold on the $m$-dimensional manifold $M$. We will show that $\mu^{A}$ is a stationary $m$-varifold, and then the Constancy Theorem [7, 41.2(3)] implies that $\mu^{A}$ is a multiple of the Riemannian volume measure $\operatorname{vol}_{M}$ as claimed. Denote the measure $\left(\pi_{2}\right)_{\#} \mu_{V}$ on $B$ by $\mu_{V, 2}$, where $\pi_{2}: M \times B \rightarrow B$ denotes the usual projection to the second component. We choose a sequence $f_{n} \in C_{c}^{\infty}(B)$ converging to the indicator function $\chi_{A}$ in $L^{1}\left(\mu_{V, 2}\right)$. This implies that $f_{n} \circ \pi_{2}$ converges to $\chi_{M \times A}$ in $L^{1}\left(\mu_{V}\right)$. Denoting the projection $M \times B \rightarrow M$ by $\pi_{1}$ we calculate for every vectorfield $X$ on $M$

$$
\begin{aligned}
\int_{M} \operatorname{div}_{M} X d \mu^{A} & =\int_{M \times A} \operatorname{div}_{M} X \circ \pi_{1} d \mu_{V} \\
& =\lim _{n \rightarrow \infty} \int_{M \times B}\left(f_{n} \circ \pi_{2}\right) \cdot\left(\operatorname{div}_{M} X \circ \pi_{1}\right) d \mu_{V} \\
& =\lim _{n \rightarrow \infty} \int_{M \times B} \operatorname{div}_{T_{p}} M \times T_{q} \mathcal{F}\left(\left(f_{n} \circ \pi_{2}\right) \cdot\left(X \circ \pi_{1}\right)\right) d \mu_{V}(p, q),
\end{aligned}
$$

since it follows from equation (6) that

$$
\operatorname{div}_{T_{p} M \times T_{q} \mathcal{F}}\left(\left(f_{n} \circ \pi_{2}\right) \cdot\left(X \circ \pi_{1}\right)\right)=\left.f_{n}(q) \cdot \operatorname{div}_{M} X\right|_{p}
$$

Since $V$ is stationary, we know from $\operatorname{Step} 2$ that $\operatorname{spt}(V) \subset \tilde{\mathcal{F}}$. Hence

$$
\delta V\left(\left(f_{n} \circ \pi_{2}\right) \cdot\left(X \circ \pi_{1}\right)\right)=\int_{M \times \mathbb{T}^{2}} \operatorname{div}_{T_{p} M \times T_{q} \mathcal{F}}\left(\left(f_{n} \circ \pi_{2}\right) \cdot\left(X \circ \pi_{1}\right)\right) d \mu_{V}(p, q)=0
$$

for all $n \in \mathbb{N}$. Thus $\int_{M} \operatorname{div}_{M} X d \mu^{A}=0$ for every vectorfield $X$ on M, i.e. the $m$ varifold defined by $\mu^{A}$ is stationary, and hence a multiple of $\operatorname{vol}_{M}$, see [7, 41.2(3)]. Using the abbreviation $\mu_{V, 2}=\left(\pi_{2}\right)_{\#} \mu_{V}$ introduced above, the constant $c_{A}$ can be calculated as follows

$$
c_{A}=\frac{1}{\operatorname{vol}_{M}(M)} \mu^{A}(M)=\frac{1}{\operatorname{vol}_{M}(M)} \mu_{V, 2}(A) .
$$

Hence, $\mu_{V}$ is given as a product of $\operatorname{vol}_{M}$ and $\mu_{V, 2}$. Next, we prove that - up to scale $-\mu_{V, 2}$ coincides with the Riemannian area $\operatorname{vol}_{\mathbb{T}^{2}}$.

The idea is to show invariance of $\mu_{V, 2}$ under the flow $\varphi_{t}^{\bar{Y}}$ of $\bar{Y}$. Then the unique ergodicity of $\varphi_{t}^{\bar{Y}}$ implies that $\mu_{V, 2}$ is a multiple of $\operatorname{vol}_{\mathbb{T}^{2}}$, cf. Corollary 1 .

We consider $f \in C^{1}(B)$ and $\tilde{X}=(f \bar{Y}) \circ \pi_{2}$. Since $\tilde{X}$ is defined in a neighborhood of $\operatorname{spt}\left(\mu_{V}\right)$ and $V$ is stationary we have

$$
0=\delta V(\tilde{X})=\int \operatorname{div}_{S} \tilde{X} d V(S)
$$


Since $\operatorname{spt}(V) \subset \tilde{\mathcal{F}}$, equation (6) implies

$$
\begin{aligned}
0 & =\int_{M \times \mathbb{T}^{2}} \operatorname{div}_{T_{p} M \times T_{q} \mathcal{F}} \tilde{X} d \mu_{V}(p, q)=\int_{M \times \mathbb{T}^{2}} g\left(\nabla_{\bar{Y}} f \bar{Y}, \bar{Y}\right) \circ \pi_{2} d \mu_{V} \\
& =\int_{M \times \mathbb{T}^{2}}\left(d f(\bar{Y})+f g\left(\nabla_{\bar{Y}} \bar{Y}, \bar{Y}\right)\right) \circ \pi_{2} d \mu_{V} \\
& =\int_{\mathbb{T}^{2}} d f(\bar{Y}) d \mu_{V, 2} .
\end{aligned}
$$

Since every function $f \in C^{1}\left(\mathbb{T}^{2}\right)$ can be extended to a $C^{1}$-function on $B$ we conclude that

$$
\int_{\mathbb{T}^{2}} d f(\bar{Y}) d \mu_{V, 2}=0
$$

for all $f \in C^{1}\left(\mathbb{T}^{2}\right)$. This implies that $\mu_{V, 2}$ is $\varphi^{\bar{Y}}$-invariant. For convenience, we include the simple proof. Since $\left(d\left(f \circ \varphi_{t}^{\bar{Y}}\right)\right)\left(\bar{Y}_{p}\right)=\left.\frac{d}{d t}\right|_{t} f \circ \varphi_{t}^{\bar{Y}}(p)$, we have for all $t>0$

$$
\begin{aligned}
0 & =\int_{0}^{t} \int_{\mathbb{T}^{2}} d\left(f \circ \varphi_{\tau}^{\bar{Y}}\right)(\bar{Y}) d \mu_{V, 2} d \tau \\
& =\int_{\mathbb{T}^{2}} f d\left(\varphi_{t \#}^{\bar{Y}}\left(\mu_{V, 2}\right)\right)-\int_{\mathbb{T}^{2}} f d \mu_{V, 2} .
\end{aligned}
$$

This together with the Borel regularity of $\mu_{V, 2}$ implies the $\varphi_{t}^{\bar{Y}}$-invariance of $\mu_{V, 2}$. Now the unique ergodicity of $\varphi_{t}^{\bar{Y}}$ implies our claim, cf. Corollary 1 .

This completes the proof of Step 3. Together, Step 2 und Step 3 prove the claimed uniqueness of $V_{0}$.

Remark 4. Actually, the calculation in Step 1 can be replaced by the following more involved argument showing that $V_{0}$ is stationary. Since $\tilde{M}$ does not satisfy an isoperimetric inequality for $(m+1)$-varifolds, B. White's Theorem 2.3 from [8] implies that $\tilde{M}$ contains a nonzero, stationary $(m+1)$-varifold $V$. But now the preceding two steps show that this $V$ is a nonzero multiple of $V_{0}$. Hence $V_{0}$ is stationary.

\section{Appendix}

Lemma 1. There is a function $R \in C^{\infty}(] 0,2[\times] 0, \pi / 2\left[, \mathbb{R}^{+}\right)$that fulfills conditions (h1) $-($ (h)

Proof. It is easy to find a function $k \in C^{\infty}\left(\mathbb{R}^{2}, \mathbb{R}\right)$ that meets conditions (R/2) and (R/3), and the following weakening of condition (R/1)

(R1') $k(1, \pi / 4)=\cos ^{2}(\pi / 4)+\alpha^{2} \sin ^{2}(\pi / 4)$.

For example $k(\rho, \psi)=(\psi-\pi / 4)^{2} \rho+(\rho-1)^{3}+\cos ^{2}(\pi / 4)+\alpha^{2} \sin ^{2}(\pi / 4)$ has these properties, but we do not need the explicit formula. In addition, we define the function $l \in C^{\infty}\left(\mathbb{R}^{2}, \mathbb{R}^{+}\right)$by $l(\rho, \psi)=\rho^{2}\left(\cos ^{2} \psi+\alpha^{2} \sin ^{2} \psi\right)$. Then

$$
(k-l)(1, \pi / 4)=0 \quad \text { and } \quad \frac{\partial}{\partial \rho}(k-l)(1, \pi / 4)=-2\left(\cos ^{2}(\pi / 4)+\alpha^{2} \sin ^{2}(\pi / 4)\right)<0 .
$$


Therefore we can find $1 / 2<\rho_{1}<\rho_{2}<1<\rho_{3}<\rho_{4}<3 / 2$ and $\pi / 8<\psi_{1}<\pi / 4<$ $\psi_{2}<3 \pi / 8$ such that for any $\psi \in\left[\psi_{1}, \psi_{2}\right]$

$$
\begin{array}{ll}
(k-l)(\rho, \psi)>0 & \text { if } \rho \in\left[\rho_{1}, \rho_{2}\right], \\
(k-l)(\rho, \psi)<0 & \text { if } \rho \in\left[\rho_{3}, \rho_{4}\right] .
\end{array}
$$

Now choose a bump function $\beta \in C^{\infty}\left(\mathbb{R}^{2},[0,1]\right)$ with support in $\left[\rho_{1}, \rho_{4}\right] \times\left[\psi_{1}, \psi_{2}\right]$, that is constantly equal to 1 in a neighbourhood of $(1, \pi / 4)$, and has the following property for any $\psi \in\left[\psi_{1}, \psi_{2}\right]$

$$
\frac{\partial}{\partial \rho} \beta(\rho, \psi)\left\{\begin{array}{ll}
\geq 0 & \text { for } \rho \in\left[\rho_{1}, \rho_{2}\right] \\
=0 & \text { for } \rho \in\left[\rho_{2}, \rho_{3}\right] \\
\leq 0 & \text { for } \rho \in\left[\rho_{3}, \rho_{4}\right]
\end{array} .\right.
$$

Since $(k-l)(1, \pi / 4)=0$ we can choose the parameters $\rho_{1}, \rho_{2}, \rho_{3}, \rho_{4}, \psi_{1}$ and $\psi_{2}$ in such a way that the function

$$
R:=(1-\beta) l+\beta k=l+\beta(k-l)
$$

is positive on the open set $] 0,2[\times] 0, \pi / 2[$. Obviously the restriction of $R$ to $] 0,2[\times] 0, \pi / 2[$ meets conditions (R/1) and (R/3). To finish the proof we check the monotonicity condition $(\mathrm{R} 2)$ :

$$
\frac{\partial}{\partial \rho} R=(1-\beta) \frac{\partial}{\partial \rho} l+\beta \frac{\partial}{\partial \rho} k+(k-l) \frac{\partial}{\partial \rho} \beta,
$$

where the sum of the first two terms is positive if $(\rho, \psi) \neq(1, \pi / 4)$ and the last term is nonnegative as (7) and (8) show.

Acknowledgements: We thank Eugene Gutkin and Stéphane Sabourau for useful comments. This work was partially supported by the DFG Collaborative Research Center SFB TR 71.

\section{REFERENCES}

1. Victor Bangert, On the existence of closed geodesics on two-spheres, Internat. J. Math. 4 (1993), no. 1, 1-10. MR MR1209957 (94d:58036)

2. George D. Birkhoff, Dynamical systems, American Mathematical Society Colloquium Publications, Vol. VIII, American Mathematical Society, Providence, R.I., 1927.

3. Tobias H. Colding and William P. Minicozzi, II, Minimal surfaces, Courant Lecture Notes in Mathematics, vol. 4, New York University Courant Institute of Mathematical Sciences, New York, 1999. MR MR1683966 (2002b:49072)

4. I. P. Cornfeld, S. V. Fomin, and Ya. G. Sinaŭ, Ergodic theory, Grundlehren der Mathematischen Wissenschaften [Fundamental Principles of Mathematical Sciences], vol. 245, Springer-Verlag, New York, 1982, Translated from the Russian by A. B. Sosinskiu. MR 832433 (87f:28019)

5. John Franks, Geodesics on $S^{2}$ and periodic points of annulus homeomorphisms, Invent. Math. 108 (1992), no. 2, 403-418. MR MR1161099 (93f:58192)

6. Peter Petersen, Riemannian geometry, Graduate Texts in Mathematics, vol. 171, SpringerVerlag, New York, 1998. MR MR1480173 (98m:53001)

7. Leon Simon, Lectures on geometric measure theory, Proceedings of the Centre for Mathematical Analysis, Australian National University, vol. 3, Australian National University Centre for Mathematical Analysis, Canberra, 1983. MR 756417 (87a:49001)

8. Brian White, Which ambient spaces admit isoperimetric inequalities for submanifolds?, J. Differential Geom. 83 (2009), no. 1, 213-228. MR MR2545035

Mathematisches Institut, Abteilung für Reine Mathematik, Albert-Ludwigs-Universität, Eckerstr. 1, 79104 Freiburg im Breisgau, Germany 